\title{
Semiclassical approach for the evaporating black hole revisited
}

\author{
Yongwan Gim* and Wontae Kim丹 \\ Department of Physics, Sogang University, Seoul 121-742, Republic of Korea
}

\begin{abstract}
A recent calculation shows that the observed energy density in the Unruh state at the future event horizon as seen by a freely falling observer is finite if the observer is released from rest at any positive distance outside the horizon; however, it is getting larger and larger so that it is negatively divergent at the horizon in the limit that the observer starts falling from rest at the horizon, which corresponds to the infinite boost with respect to the freely falling observer at a finite distance from the horizon. In order to resolve some conflicts between the recent calculation and the conventional ones in the well-known literatures, the calculation of the free-fall energy density is revisited and some differences are pointed out.
\end{abstract}

Keywords: free falling, Hawking radiation, Unruh state

\footnotetext{
${ }^{*}$ Email: yongwan89@sogang.ac.kr

†Email: wtkim@sogang.ac.kr
} 


\section{1 introduction}

One of the most outstanding works over the last four decades in the quantum theory of gravity is the derivation of Hawking radiation from black holes [1] since it has provided many profound questions and intriguing puzzles about the quantum theory of gravity such as information loss problem [2], black hole complementarity [3, 4, 5], and the recent firewall paradox [6, 7] which states that a freely falling observer when crossing the horizon encounters firewalls which are high frequency outgoing quanta near the horizon and the infalling observer burns up [6, 7]. Subsequently, various aspects related to the firewall issue have been extensively studied in Refs. [8, 9, 10, 11, 12, 13, 14, 15, 16. The presence of the firewalls has something to do with the failure of the equivalence principle or breakdown of semiclassical physics at macroscopic distance from the horizon, which eventually makes black hole complementarity incomplete. A similar prediction referred to as an energetic curtain has also been done based on different assumptions [17]. On the other hand, it has been claimed that firewalls are not essential since the unitary evolution of black hole entangles a late mode located outside the event horizon with a combination of early radiation and black hole states at the same time [18], and also argued that the remaining set of nonsingular realistic states do not have firewalls but yet preserve information [19]. Of course, it should be emphasized that a static outside observer would not witness formation of the horizon and always sees unitary evolution [20] without any firewall-like object. Since all these issues are related to the freely falling observer, the revisit of the free-fall energy density becomes so important. Note that the classic work [21] tells us that the energy density in the Unruh state [22] and the Israel-Hartle-Hawking state [23, 24] are finite on the future horizon, although it is divergent in the Boulware state [25] on the future horizon. Especially, for the black hole in the Unruh state, the energy-momentum tensors were calculated at the bifurcation two-sphere in virtue of the vanishing affine connections since the energy-momentum tensors could be regarded as quantities in the locally flat spacetimes [21]. This calculation was in turn extended to the future horizon by taking into account of a symmetry argument for the infinite time, and the finite energy density was eventually obtained on the future horizon when the observer is dropped at the future event horizon without any journey. The geodesic solution was not used in this work so that the conclusion seems to be dubious if the employed coordinates are not the local free-fall coordinates. On the other hand, quantum gravitational collapse was investigated from the point of view of an infalling observer in order to investigate the quantum-mechanical modification of the collapse and the singularity [26]. And it was also pointed out that the observers dropped from a finite distance outside 
the horizon will detect a finite amount of radiation when crossing the horizon by using the effective temperature method [27, 28]. Recently, the free-fall energy density in the soluble two-dimensional Schwarzschild black hole [29] was directly calculated at arbitrary free-fall positions in order to study the dependence of initial free-fall positions of the free-fall energy density on the horizon and clarify whether the freely falling observer could encounter something non-trivial effect at the horizon or not. In particular, solving the geodesic equation of motion exactly over the whole region outside the horizon in the Unruh state [22, it could be shown that there exists the negative energy density up to the extent to the exterior to the horizon of the black hole, roughly $r \sim 3 M$ [29], where the similar argument in connection with the firewall argument appears in Ref. [30] and the behavior of the energy density was also explicitly shown in Ref. [31]. Note that the negative energy density is getting larger and larger when the initial infalling position from being at rest approaches the horizon [29]. So, the energy density in the freely falling frame is finite unless the observer is not dropped at the horizon. However, if the observer were dropped at the horizon, the energy-density would be divergent. So, one might wonder why the behavior of the energy density in the freely falling frame at the horizon in Refs. [29] is different from the result in Ref. [21.

In this work, we would like to elaborate what the differences between the finite energy density calculated on the future horizon [21] and the recent calculations for the divergent energy density on the future horizon [29]. Usually, the Kruskal coordinates might be used in calculating the free-fall energy density on the horizons; however, in Sec. 2, we shall prove that affine connections derived in the Kruskal coordinates do not always vanish at $r=2 M$, specifically along the ingoing direction on the future horizon. It means that it is impossible to regard the energy density calculated in the Kruskal coordinates as the one observed in the freely falling frame, since the Kruskal coordinates do not play a role of the local inertial coordinates any more, so that the Kruskal time is no longer proper time on the future horizon. Thus we should consider the general coordinate transformation of the energy-momentum tensors from the fixed coordinates such as the Kruskal coordinates to the local inertial coordinates, which gives the appropriate definition for infalling energy density observed in the local inertial frame [32] in Sec. 3. After all, we will show that the observed energy density in the Unruh state at the future event horizon as seen from a freely infalling observer is finite if the observer is released from rest at any positive distance outside the horizon, where the result is compatible with the one in Refs. [27, 28]; however, it is only divergent at the horizon in the limit that the freely falling observer starts falling from rest at the horizon [29]. In Sec. 4, the origin of the divergence will also be mentioned from the viewpoint of the infinite blue shift which is related to the infinite boost with respect 
to relatively infalling observer at a finite distance from the horizon. Finally, the conclusion and comment will be presented in Sec. 5 .

\section{Kruskal coordinates}

To explain the reason why the different behavior of the energy density appears between the recent calculation [29] and the classic work [21] with the well-known text book [34], we would like to present a heuristic calculation in terms of the Callan-Giddings-Harvey-Strominger model [33], where the length element is given as $d s^{2}=-e^{2 \rho} d x^{+} d x^{-}$together with the metric component of $e^{-2 \rho}=M / \lambda-\lambda^{2} x^{+} x^{-}$in the Kruskal coordinates. The Kruskal coordinates are related to the tortoise coordinates through the coordinate transformations of $2 \lambda t=$ $\ln \left(-x^{+} / x^{-}\right)$and $2 \lambda r^{*}=\ln \left(-\lambda^{2} x^{+} x^{-}\right)$, where $r^{*}=r+(1 / 2 \lambda) \ln \left[1-(M / \lambda) e^{-2 \lambda r}\right]$. The affine connections in the Kruskal coordinates are straightforwardly calculated as $\Gamma_{++}^{+}\left(x^{+}, x^{-}\right)=$ $2 \partial_{+} \rho\left(x^{+}, x^{-}\right) \sim x^{-}, \Gamma_{--}^{-}\left(x^{+}, x^{-}\right)=2 \partial_{-} \rho\left(x^{+}, x^{-}\right) \sim x^{+}$. Note that the affine connection of $\Gamma_{--}^{-}\left(x^{+}, 0\right)$ on the future horizon of $x^{-}=0$ does not vanish, while $\Gamma_{++}^{+}\left(x^{+}, 0\right)=0$. So, the geodesic equation of motion tells us that $x^{-}$cannot be a local flat coordinate on the future horizon. However, the affine connections vanish at $x^{ \pm}=0$ corresponding to the bifurcation point.

The awkward situation is not restricted to the above case, and it also happens in the other models such as the two-dimensional Schwarzschild black hole which is actually of our concern since the model is simple but it shares most properties in realistic four-dimensional black holes. The length element is given as $d s^{2}=-f(r) d t^{2}+f^{-1}(r) d r^{2}$ with the metric function of $f(r)=1-2 M / r$ in the Schwarzschild coordinates. The conformal factor for the length element of $d s^{2}=-e^{2 \rho} d x^{+} d x^{-}$in the Kruskal coordinates is obtained as

$$
e^{2 \rho\left(x^{+}, x^{-}\right)}=\frac{2 M}{r} e^{1-\frac{r}{2 M}}
$$

from the conformal transformation of $x^{ \pm}= \pm 4 M e^{ \pm \sigma^{ \pm} / 4 M}$, where $\sigma^{ \pm}=t \pm r^{*}$ and $r^{*}=r-$ $2 M+2 M \ln (r / 2 M-1)$. The corresponding coordinate transformations are implemented by $t=2 M \ln \left(-x^{+} / x^{-}\right)$and $r^{*}=2 M \ln \left(-x^{+} x^{-} /\left(16 M^{2}\right)\right)$. At first glance, the affine connections calculated from Eq. (11) might be expected to vanish at $r=2 M$ since it is given as

$$
\Gamma_{ \pm \pm}^{ \pm}(t, r)=\mp\left(\frac{1}{2 r}+\frac{M}{r^{2}}\right) \sqrt{\frac{r}{2 M}-1} e^{\nexists t-r+2 M}
$$

in the Kruskal coordinates. But this is not the case except the finite $t$ since the vanishing square root and the divergent exponential function in $\Gamma_{--}^{-}$compete on the future horizon. So, it might be tempted that the affine connections at $t \rightarrow \infty$ would vanish on the future 
horizon away from the bifurcation two-sphere. However, this is not the case as seen from Eqs. (5) and (66), and hence the two limits such as $r=2 M$ and $t \rightarrow \infty$ should be taken at one stroke in order to justify the flatness via affine connections on the future horizon. For this purpose, if we take advantage of the light cone expressions in the Kruskal coordinates, then the affine connections (2) are neatly calculated as

$$
\begin{aligned}
& \Gamma_{++}^{+}\left(x^{+}, x^{-}\right)=\frac{1}{x^{+}}\left(\frac{1}{(1+W(Z))^{2}}-1\right), \\
& \Gamma_{--}^{-}\left(x^{+}, x^{-}\right)=\frac{1}{x^{-}}\left(\frac{1}{(1+W(Z))^{2}}-1\right),
\end{aligned}
$$

in virtue of the Lambert $W$ function defined as $Z=W(Z) e^{W(Z)}$ where $Z=-x^{+} x^{-} /\left(16 M^{2}\right)$. As a result, the affine connections on the future horizon of $x^{-}=0$ are written as

$$
\begin{aligned}
& \lim _{x^{-} \rightarrow 0} \Gamma_{++}^{+}\left(x^{+}, x^{-}\right)=0, \\
& \lim _{x^{-} \rightarrow 0} \Gamma_{--}^{-}\left(x^{+}, x^{-}\right)=\frac{x^{+}}{8 M^{2}} \neq 0,
\end{aligned}
$$

where we used the relation of $W(Z)=Z-Z^{2}-O\left(Z^{3}\right)$ near the future horizon. Note that $\Gamma_{--}^{-}$does not vanish on the future horizon, and it turns out that the coordinate $x^{-}$cannot be a freely falling coordinate.

As expected, these two-dimensional results can also be applied to the four-dimensional Schwarzschild metric whose length element is given as $d s^{2}=-e^{2 \rho} d x^{+} d x^{-}+r^{2}\left(d \theta^{2}+\right.$ $\sin ^{2} \theta d \phi^{2}$ ), where $\rho$ and $r$ are functions of $x^{ \pm}$. The corresponding nonvanishing affine connections on the future horizon are illustrated such as $\Gamma_{--}^{-}=x^{+} /\left(8 M^{2}\right), \Gamma_{\theta \theta}^{+}=-x^{+} / 2, \Gamma_{\phi \phi}^{+}=$ $(-1 / 2) x^{+} \sin ^{2} \theta, \Gamma_{-\theta}^{\theta}=-x^{+} /\left(16 M^{2}\right), \Gamma_{\phi \phi}^{\theta}=-\cos \theta \sin \theta, \Gamma_{-\phi}^{\phi}=-x^{+} /\left(16 M^{2}\right)$, and $\Gamma_{\theta \phi}^{\phi}=$ $\cot \theta$. We can choose $\theta=\pi / 2$ since we are concerned with the freely falling motion which is confined on the plane, but there still exist nonvanishing affine connections on the future horizon. In the light of these calculations, the Kruskal coordinates could not be local flat coordinates on the future horizon except the bifurcation point joining the past horizon and the future horizon corresponding to $x^{ \pm}=0$. Note that in Ref. [21], the energy-momentum tensors were calculated on the bifurcation two-sphere for which $\Gamma_{ \pm \pm}^{ \pm}(t, 2 M)=0$ for any finite time, and in turn extended the analysis to the future horizon by taking infinite time with a symmetry argument without any coordinate transformation to the local inertial frames. Thus, if the energy-momentum tensors were calculated in the Kruskal coordinates on the future horizon, they could not be identified with the energy momentum tensors in the freely falling frame at that point. So the finiteness of the energy momentum tensors at the future event horizon should be reexamined. 


\section{3 energy density in freely falling frame}

At the asymptotic infinity, the energy-momentum tensors are easily defined in virtue of the tortoise coordinates since they are more amenable to impose some boundary conditions compared to the other coordinate systems. Let us now consider the energy-momentum tensors in the tortoise coordinates, and assume that the tensor transformations can be welldefined from the tortoise coordinates to the Kruskal coordinates as a true tensors without any anomalies,

$$
\begin{aligned}
& T_{ \pm \pm}\left(x^{+}, x^{-}\right)=\left(\frac{\partial \sigma^{ \pm}}{\partial x^{ \pm}}\right)^{2} T_{ \pm \pm}\left(\sigma^{+}, \sigma^{-}\right), \\
& T_{+-}\left(x^{+}, x^{-}\right)=\left(\frac{\partial \sigma^{+}}{\partial x^{+}}\right)\left(\frac{\partial \sigma^{-}}{\partial x^{-}}\right) T_{+-}\left(\sigma^{+}, \sigma^{-}\right) .
\end{aligned}
$$

If the energy-momentum tensors were calculated in the Kruskal coordinates, they could not be identified with those observed by the freely falling observer except the bifurcation point as discussed in the previous section. Thus the coordinates $x^{ \pm}$should be replaced by the local inertial coordinates in such a way that the energy density in the local inertial coordinate should be written as

$$
\epsilon=T_{\tau \tau}=\frac{d \sigma^{\mu}}{d \tau} \frac{d \sigma^{\nu}}{d \tau} T_{\mu \nu}
$$

where $\tau$ is a proper time [32]. In other words, the energy-momentum tensors (7) and (8) calculated in the Kruskal coordinates should be transformed to the local inertial coordinates. Note that such a form of the energy density (9) was already introduced in order to exhibit the finite infalling energy density on the future horizon in Ref. [34]. The authors considered an observer moving along a line of constant Kruskal position of $x^{1}=a$ where $a$ is a constant along with the two-velocity of $\left(u^{0}, u^{1}\right)=\left(d x^{0} / d \tau, d x^{1} / d \tau\right)=e^{-\rho}(1,0)$. The constant spacial radius was expressed in the light cone coordinates as $x^{+}=x^{-}+2 a$ in the Kruskal coordinates. However, the constant line does not obey the geodesic equation of motion but it can be a geodesic solution only at the bifurcation point for which $a=0$. Thus the calculation does not warrant the finiteness of the energy density in the freely falling frame at the future horizon even in spite of the correct definition of the infalling energy density (9) 1 .

Now, it becomes clear why we have to use the above definition of the infalling energy density along with the correct geodesic solution in order to study the energy density in the

\footnotetext{
${ }^{1}$ We have repeatedly been asked why our result in this work is incompatible with the result of Ref. 34. in the well-known text book. Our answer is: "The proposed geodesic curve in section 8.2 does not actually satisfy the geodesic equation of motion, so that the energy density by the freely falling observer discussed in Ref. [34] is not the free-fall energy density".
} 
freely falling frame. Using Eq. (9), we are going to calculate the infalling energy density in the two-dimensional Schwarzschild black hole in the Unruh state by means of the light-cone coordinates in order to avoid any ambiguities in connection with the future horizon. Let us now start with the conformal gauge fixed energy-momentum tensors [35],

$$
\begin{aligned}
& T_{ \pm \pm}=-\kappa\left[\left(\partial_{ \pm} \rho\right)^{2}-\partial_{ \pm}^{2} \rho+t_{ \pm}\right] \\
& T_{+-}=-\kappa \partial_{+} \partial_{-} \rho,
\end{aligned}
$$

where they can be derived from the covariant conservation law and the two-dimensional trace anomaly for the number of $N$ massless scalar fields and $t_{ \pm}$are the integration functions and $\kappa=N / 12$. The conformal factor of the two-dimensional Schwarzschild black hole from Eq. (1) is written as

$$
e^{2 \rho\left(\sigma^{+}, \sigma^{-}\right)}=1-\frac{2 M}{r\left(\sigma^{+}, \sigma^{-}\right)},
$$

in terms of the tortoise coordinates, where the radial coordinate is also expressed as $r\left(\sigma^{+}, \sigma^{-}\right)=$ $2 M(1+W(Y))$ and by definition $Y=\exp \left[\left(\sigma^{+}-\sigma^{-}\right) / 4 M\right]$. From Eqs. (10), (11) and (12), it is easy to obtain the energy momentum tensors,

$$
\begin{aligned}
& T_{++}=-\frac{\kappa}{64 M^{2}} \frac{1+4 W(Y)}{(1+W(Y))^{4}}, \\
& T_{--}=-\frac{\kappa}{64 M^{2}}\left(\frac{1+4 W(Y)}{(1+W(Y))^{4}}-1\right), \\
& T_{+-}=\frac{\kappa}{16 M^{2}} \frac{W(Y)}{(1+W(Y))^{4}},
\end{aligned}
$$

which satisfy the Unruh state because we chose $t_{+}=0$ and $t_{-}=-1 /\left(64 M^{2}\right)$ [22]. So, the ingoing flux is negative finite on the past horizon from Eq. (13) and hence it is singular in the Kruskal coordinates, while there is no outgoing flux on the future horizon from Eq. (14) so that it is finite in the Kruskal coordinate on the future horizon.

Next, the components of the two-velocity are obtained by exactly solving the geodesic equation of motion for a massive particle as

$$
u^{ \pm}\left(\sigma^{+}, \sigma^{-} ; \sigma_{s}^{+}, \sigma_{s}^{-}\right)=\left(\sqrt{1-\frac{1}{1+W\left(Y_{s}\right)}} \pm \sqrt{\frac{1}{1+W(Y)}-\frac{1}{1+W\left(Y_{s}\right)}}\right)^{-1}
$$

where the initial infalling position at rest is denoted by $\sigma_{s}^{ \pm}$, and $Y_{s}=\exp \left[\left(\sigma_{s}^{+}-\sigma_{s}^{-}\right) / 4 M\right]$. From Eqs. (13),(14), (15), and (16), the energy density (9) measured on the future horizon is given as

$$
\epsilon\left(\sigma^{+}, \sigma^{-} \rightarrow \infty ; \sigma_{s}^{+}, \sigma_{s}^{-}\right)=-\frac{\kappa}{256 M^{2} W\left(Y_{s}\right)}-\frac{33 \kappa}{256 M^{2}}+O\left(W\left(Y_{s}\right)\right),
$$


where the initial infalling position is assumed to be near the future horizon. It is interesting to note that it is independent of $\sigma^{+}$, and just depends on the initial infalling position $\sigma_{s}^{ \pm}$. From Eq. (17), it turns out that there is no divergence unless we require that the observer be at rest at the horizon. On the other hand, if the observer is dropped extremely on the future horizon for which $Y_{s}$ and $W\left(Y_{s}\right)$ vanish, then the energy density is negative divergent.

\section{4 blue shift}

So far we have calculated the energy density in the freely falling frame in the Unruh state near the future horizon. Let us now discuss, in particular, the origin of the divergence when the observer is dropped at the horizon as an extreme limit. Considering a freely falling observer at the initial infalling position of $r_{s}$ without any journey for simplicity, the energy density (9) is written as $\epsilon\left(r_{s} ; r_{s}\right)=T_{t t} u^{t} u^{t}+T_{r r} u^{r} u^{r}+2 T_{t r} u^{t} u^{r}$ in the Schwarzschild coordinates. When the infalling happens at rest i.e., $\left.u^{r}\right|_{r_{s}}=0$, then the infalling energy density at that moment is reduced to $\epsilon\left(r_{s} ; r_{s}\right)=\left(1 / f\left(r_{s}\right)\right) T_{t t}$ in virtue of $\left.u^{t}\right|_{r_{s}}=d t /\left.d \tau\right|_{r_{s}}=1 / \sqrt{f\left(r_{s}\right)}$. Note that the red-shift factor is also responsible for the gravitational time dilation which is larger and larger as the initial infalling position approaches the horizon. Next, the value of $T_{t t}$ in the Schwarzschild coordinates can be directly obtained by the use of the coordinate transformation from the tortoise coordinates to the Schwarzschild coordinates, then the energy density (9) becomes

$$
\epsilon\left(r_{s} ; r_{s}\right)=\left.\frac{1}{f\left(r_{s}\right)}\left[T_{++}+T_{--}+2 T_{+-}\right]\right|_{r_{s}},
$$

where the last term is independent of the vacuum state of black hole and it can be written as $T_{+-} \sim-\left(\kappa /\left(16 M^{2}\right)\right) f\left(r_{s}\right)$ near the horizon.

When the initial infalling position extremely approaches the horizon $r_{s} \rightarrow r_{H}$, Eq. (10) can also be expanded asymptotically for each of vacuum states. First, the leading order of contributions to the energy-momentum tensors in the Boulware state described by choosing $t_{ \pm}=0$ [25] becomes finite since $T_{ \pm \pm}^{\mathrm{B}} \sim-\kappa /\left(64 M^{2}\right)$, so that the energy density (18) is divergent at the horizon. For the Israel-Hartle-Hawking state implemented by choosing $t_{+}=t_{-}=-1 /\left(64 M^{2}\right)[23$, 24], the leading order of energy-momentum tensors is written as $T_{ \pm \pm}^{\mathrm{H}} \sim-\left(\kappa /\left(16 M^{2}\right)\right) f\left(r_{s}\right)$ which vanish asymptotically at the horizon; however, the energy density is finite due to the redshift factor in the denominator in Eq. (18). Hence, these two states result in drastically different conclusions. By the way, in the Unruh state characterized by $t_{+}=0$ and $t_{-}=-1 /\left(64 M^{2}\right)$, the leading order of the energy-momentum tensors near the

horizon is calculated asymmetrically as $T_{++}^{\mathrm{U}} \sim-\kappa /\left(64 M^{2}\right)$ and $T_{--}^{\mathrm{U}} \sim-\left(\kappa /\left(16 M^{2}\right)\right) f\left(r_{s}\right)$, 
where the ingoing flux is negative finite while the outgoing one vanishes at the horizon. However, the energy density observed in the freely falling frame at the horizon is divergent because the negative finite ingoing flux $T_{++}^{\mathrm{U}}$ is infinitely blue shifted just like the case of the Boulware state. Actually, in this case, the infinite boost is required with respect to the freely falling observer from a finite distance. So the divergent effect is from moving at the speed of light relative to any infalling frame that comes from any positive distance outside the horizon. Thus the divergence is easily explained as a blueshift effect from moving at the speed of light through radiation.

\section{5 conclusions}

We showed that the Kruskal coordinates could not be local inertial coordinates on the future horizon by invoking nonvanishing affine connections. So we investigated the energy density observed by the freely falling observer in the Unruh state by means of the proper definition of the energy density. The energy density observed at the future horizon by the freely falling observer from rest is finite unless the observer is dropped at the horizon. For the extreme case of the observer dropped at the horizon, the energy density is divergent, which is due to the infinite blue shift of the energy density. The closer the initial infalling position approaches the horizon, the more negative energy density appears. Our calculations show that the energy density observed by the freely falling observer at the future horizon is sensitive to both the vacuum state of black hole and the initial infalling position of the freely falling observer. In Ref. [21], the author concluded that the energy density in the fixed coordinate at the horizon is finite without specifying the geodesic solution and initial free-fall position. And in Ref. [34] the correct definition of the energy density was used; however, the geodesic curve does not satisfy the geodesic equation of motion. Therefore, the correct geodesic trajectory and the definition of the free-fall energy density were used in this work compared to the well-known literatures.

The final comment is in order. It is expected to observe some amount of the energy density near the horizon, which amounts to the curvature scale of $\sim 1 / M^{2}$. Thus the energy density in the freely falling frame can be written as the improved form by the red shift factor as $\epsilon \sim 1 /\left[M\left(r_{s}-2 M\right)\right]$ from Eq. (18). If the observer were dropped at a finite distance but close to the horizon, for example, $\left|r_{s}-2 M\right| \ll 1 / M$, the energy density observed by the freely falling observer would be a huge amount of energy density in this region. The energy density even in the large black hole can distort the local flatness in the local inertial frame due to the contribution of the blue shift to the energy density compared to the scale of the 
black hole. This deserves further attention whether the equivalence principle is still valid or not in this special region.

\section{Acknowledgments}

We would like to thank Myungseok Eune and Edwin J. Son for exciting discussions at the first stage of this work. In particular, WK would like to thank Sam L. Braunstein, William G. Unruh, and Joseph Polchinski for helpful comments, and was greatly indebted to Don Page for many valuable comments in particular for the explicit explanation of the infinite acceleration at the horizon in section IV on this work.

\section{References}

[1] S. W. Hawking, Commun. Math. Phys. 43, 199 (1975) [Erratum-ibid. 46, 206 (1976)].

[2] S. W. Hawking, Phys. Rev. D 14, 2460 (1976).

[3] L. Susskind, L. Thorlacius and J. Uglum, Phys. Rev. D 48, 3743 (1993) arXiv:hep-th/9306069.

[4] C. R. Stephens, G. 't Hooft and B. F. Whiting, Class. Quant. Grav. 11, 621 (1994) gr-qc/9310006.

[5] L. Susskind and L. Thorlacius, Phys. Rev. D 49, 966 (1994) arXiv:hep-th/9308100.

[6] A. Almheiri, D. Marolf, J. Polchinski and J. Sully, JHEP 1302, 062 (2013) arXiv:1207.3123 [hep-th]].

[7] A. Almheiri, D. Marolf, J. Polchinski, D. Stanford and J. Sully, JHEP 1309, 018 (2013) arXiv:1304.6483 [hep-th]].

[8] S. D. Mathur, Class. Quant. Grav. 26, 224001 (2009) arXiv:0909.1038 [hep-th]].

[9] R. Bousso, Phys. Rev. D 87, 124023 (2013) arXiv:1207.5192 [hep-th]].

[10] Y. Nomura, J. Varela and S. J. Weinberg, JHEP 1303, 059 (2013) arXiv:1207.6626 [hep-th]].

[11] D. N. Page, JCAP 1304, 037 (2013) arXiv:1211.6734 [hep-th]].

[12] S. B. Giddings, Phys. Rev. D 88, 064023 (2013) arXiv:1211.7070 [hep-th]]. 
[13] T. Jacobson, Int. J. Mod. Phys. D 22, 1342002 (2013) arXiv:1212.6944 [hep-th]].

[14] W. Kim, B.-H. Lee and D.-H. Yeom, JHEP 1305, 060 (2013) [arXiv:1301.5138 [gr-qc]].

[15] J. Maldacena and L. Susskind, Fortsch. Phys. 61, 781 (2013) arXiv:1306.0533 [hep-th]].

[16] M. Hotta, J. Matsumoto and K. Funo, Phys. Rev. D 89, no. 12, 124023 (2014) arXiv:1306.5057 [quant-ph]].

[17] S. L. Braunstein, S. Pirandola and K. Zyczkowski, Phys. Rev. Lett. 110, 101301 (2013).

[18] J. Hutchinson and D. Stojkovic, arXiv:1307.5861 [hep-th].

[19] D. N. Page, JCAP 1406, 051 (2014) [arXiv:1306.0562 [hep-th]].

[20] A. Saini and D. Stojkovic, Phys. Rev. Lett. 114, no. 11, 111301 (2015) arXiv:1503.01487 [gr-qc]].

[21] P. Candelas, Phys. Rev. D 21, 2185 (1980).

[22] W. G. Unruh, Phys. Rev. D 14, 870 (1976).

[23] W. Israel, Phys. Lett. A 57, 107 (1976).

[24] J. B. Hartle and S. W. Hawking, Phys. Rev. D 13, 2188 (1976).

[25] D. G. Boulware, Phys. Rev. D 111404 (1975).

[26] E. Greenwood and D. Stojkovic, JHEP 0806, 042 (2008) doi:10.1088/11266708/2008/06/042 [arXiv:0802.4087 [gr-qc]].

[27] L. C. Barbado, C. Barcelo and L. J. Garay, Class. Quant. Grav. 28, 125021 (2011) arXiv:1101.4382 [gr-qc]].

[28] L. C. Barbado, C. Barcelo and L. J. Garay, Class. Quant. Grav. 29, 075013 (2012) arXiv:1201.3820 [gr-qc]].

[29] M. Eune, Y. Gim and W. Kim, Mod. Phys. Lett. A 29, no. 40, 1450215 (2014) arXiv:1401.3501 [hep-th]].

[30] B. Freivogel, JCAP 1407, 041 (2014) arXiv:1401.5340 [hep-th]].

[31] S. Singh and S. Chakraborty, Phys. Rev. D 90, 024011 (2014) arXiv:1404.0684 [gr-qc]]. 
[32] L. H. Ford and T. A. Roman, Phys. Rev. D 48, 776 (1993) [gr-qc/9303038].

[33] C. G. Callan, Jr., S. B. Giddings, J. A. Harvey and A. Strominger, Phys. Rev. D 45, 1005 (1992) hep-th/9111056.

[34] N.D. Birrell, P.C.W. Davies, Quantum Fields In Curved Space, Cambridge Univ. Press, Cambridge, UK, 1982.

[35] S. M. Christensen and S. A. Fulling, Phys. Rev. D 15, 2088 (1977). 